\title{
Fracking, yacimientos en roca generadora y salud humana: entre la incertidumbre y la precaución
}

\author{
Fracking, source rock-reservoir and human health: \\ between uncertainty and precaution
}

\author{
Jorge Pinto-Valderrama ${ }^{1}$; Alvaro-J Idrovo ${ }^{1}$
}

Forma de citar: Pinto-Valderrama J, Idrovo AJ. Fracking, yacimientos en roca generadora y salud humana: entre la incertidumbre y la precaución. Rev Univ Ind Santander Salud. 2019; 51(2): 99-102. doi: http://dx.doi.org/10.18273/revsal.v51n2-2019001 (c) (i)

El pasado 14 de Febrero de 2019, la Comisión ad hoc de Expertos convocada por el gobierno nacional entregó su informe, en donde conceptuó avalando la posibilidad de explotar yacimientos no convencionales en Colombia y recomendó avanzar con las pruebas piloto de perforación, pruebas que hacen uso de una técnica denominada fracking (en español fracturación hidráulica). Ante esta realidad inminente es necesario que los profesionales de la salud conozcan del tema, en especial aquellos que laboran en las regiones donde existe un alto potencial de hidrocarburos que requieren el uso de esta tecnología para su explotación, como son Santander (con el 56\% de la roca de interés), Cesar (con un 28\%), Bolivar (con 8\%) y Antioquia (con el 7\%), y en menor proporción Boyacá, Cundinamarca, Norte de Santander y Tolima.

El fracking es una tecnología usada con diferetes propósitos en la industria de los hidrocarburos que en su esencia más básica, consiste en inducir fisuras en la roca inyectando agua a presión (90\%), junto a una serie de aditivos $(0,5 \%)$, a través de las paredes del pozo y mantener estas fisuras abiertas introduciendo arena $(9,5 \%)$ en ellas, para así mejorar el paso del hidrocarburo líquido y gaseoso hacia la superficie. Esta técnica ha sido ampliamente usada en Colombia con éxito para la explotación de campos petroleros de crudos pesados, y también para la revitalización de campos maduros ${ }^{1,2}$ que ya estaban declinando su producción, como Cusiana y Cupiagua, ambos en la Orinoquia colombiana. Sin embargo, estos usos de la técnica no habían despertado tanta preocupación y debate en la sociedad como su implementación para explotar Yacimientos No Convencionales (YNC).

Existen varios tipos de YNC, y entre aquellos que requieren fracturamiento hidráulico para su explotación se encuentran el "gas shale" y el "oil shale" que, en español, los podríamos agrupar como "Yacimientos en Roca Generadora" (YRG). La naturaleza geológica de estos yacimientos y la ingeniería aplicada para su aprovechamiento es más compleja que la de los yacimientos tradicionalmente explotados en Colombia desde hace cien años; primero, por la presencia de elementos radioactivos y gases tóxicos en la roca, que tienen una alta capacidad de migración, y segundo, por las características propias de las técnicas usadas en la fase de perforación, en donde se hace uso de químicos especiales, grandes volúmenes de agua y una alta densidad de perforaciones. Si bien, estos dos factores han sido tenidos en cuenta por los países para tomar la decisión de si hacen o no fracking para aprovechar los YRG, es importante citar que la controversia es amplia por cuanto diversos países han tomado posturas igualmente diversas, como resultado de sus políticas ambientales y de salud ambiental.

Este tipo de yacimientos en años recientes ha incrementado su uso en varios países, como Canadá, la mayor parte de los estados de Estados Unidos de América, Argentina, China y desde hace unas semanas, Inglaterra en el Reino Unido. Esto contrasta con países donde su explotación se encuentra en moratoria hasta tener estudios concluyentes, como Alemania, Rusia y Escocia. ${ }^{3}$ En Francia, Bélgica, y parte de Australia al igual que en algunos estados de la

1. Universidad Industrial de Santander. Bucaramanga, Colombia. 
Unión Americana (New York) se ha prohibido por sus riesgos. En Colombia la decisión política es hacer fracking para incrementar sustancialmente las reservas de petróleo y gas, siguiendo el camino marcado por Estados Unidos de América. Teniendo ya más claro qué es el fracking, es importante conocer la evidencia que lo relaciona con salud humana. A continuación se listarán algunos de estos temas.

Inducción de sismos. La evidencia señala que si bien el fracturamiento hidráulico como tal no es el causante de los $\operatorname{sismos}^{1}$, la práctica de reinyectar el agua extraída del subsuelo en forma contínua y acelerada si lo es, produciendo microsismos (Richter entre 1 y 2), sismos menores y de baja magnitud (Richter 3 y 4). El sismo de mayor magnitud que se ha registrado hasta el momento fue de 4,4 en la escala de Richter (centro-oeste de Alberta y noreste de British Columbia $^{2}$ ), producido al reactivar fallas preexistentes ${ }^{4,5}$, por lo que resulta claro que la reinyección no debería realizarse cerca a fallas geológicas y además, que se requiere un permanente monitoreo de las áreas en donde se desarrolle tal actividad ${ }^{6}$.

Ruido y miedo por el fracking. Residentes en lugares cercanos a lugares donde se realiza el fracking se quejan del ruido, que puede llegar a 61 decibeles, generando ansiedad y trastornos del sueño ${ }^{7}$ con el consecuente deterioro de la salud mental.

Efectos tóxicos del fracking. Las mezclas químicas usadas como aditivos en el fracking pueden ser tóxicas para el ser humano ${ }^{8,9}$, tal cual se indica por análisis de los 105 aditivos más usados en Canadá ${ }^{10}$. Si bien como se citó al inicio, las concentraciones usadas para los aditivos son bajas, y al momento de iniciar su tránsito por la roca se diluyen hasta alcanzar concentraciones permitidas por la ley, es importante controlar que no se autorice el uso en Colombia de los aditivos que por su toxicidad ya han sido prohibidos en otras latitudes. En los últimos diez años estos aditivos se han constituido en el principal foco de debate respecto a la técnica por sus efectos en la salud humana y el medio ambiente ${ }^{3}$, generando estudios que llevaron a la creación de normas rigurosas, en donde se prohibe el uso de algunos químicos y se establecen concentraciones máximas para otros. De esta manera, las mezclas químicas pueden llegar al humano por inhalación aérea, especialmente a los pobladores más cercanos al sitio de perforación en superficie.

Contaminación de fuentes hídricas y suelos. La probabilidad de que un agente tóxico escape por las paredes del pozo y migre desde profundidades cercanas a los 3 kilómetros, asociado a los gases liberados de la roca fracturada, es realmente muy baja, debido a la rigurosa normatividad que se ha aprobado en Colombia recogiendo la regulación más estricta que se ha implementado en otras partes del mundo. Sin embargo, deberíamos estudiar la eficiencia y efectividad de estos controles, estableciendo un nivel base ambiental y social previo a las pruebas piloto y siguiendo activamente sus cambios en el tiempo.

Es más probable que pueda producirse contaminación del suelo y el agua por una mala práctica en la implementación de los protocolos industriales en superficie, y si este fuera el caso, la exposición humana a agentes tóxicos puede llegar por diversas vías, principalmente la ingesta. Al respecto, la Agencia de Protección Ambiental (EPA, por sus siglas en inglés) de Estados Unidos de América señala seis posibles formas en que los acuíferos podrían verse afectados: al hacer uso industrial del agua en zonas o épocas del año con baja disponibilidad del fluído, por derrames durante el transporte y manejo en superficie de fluidos o químicos empleados en fracturamiento hidraúlico en volúmenes o concentraciones grandes, por hacer inyección de fluidos para fracturamiento hidraúlico en pozos con integridad comprometida que permita filtraciones; o la descarga de aguas residuales tratados de manera inadeacuada en escorrentías, menos probable, por la inyección de fluídos para fracturamiento hidraúlico directamente en acuíferos y, finalmente, por almacenamiento o disposición de fluidos o aguas residuales del fracturamiento hidraúlico en piscinas con revestimientos inapropiados que permitan filtraciones.

El tema de las mezclas químicas es complejo, porque los efectos tóxicos no son conocidos, la migración de los gases depende de la porosidad y permeabilidad propia de cada lugar, y la variedad de estos, no debe reducirse a la

1 https://earthquake.usgs.gov/research/induced/myths.php . Induced Earthquakes: Myths and Misconceptions. USGS. 2017.

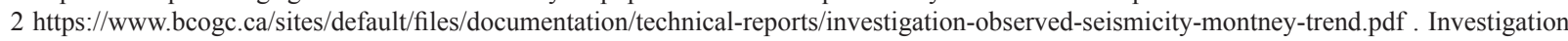
of Observed Seismicity in the Montney Trend. BC Oil and Gas Commission. BCOGC. 2014.

$3 \mathrm{https} / / /$ www.epa.gov/hfstudy. EPA's Study of Hydraulic Fracturing for Oil and Gas and Its Potential Impact on Drinking Water Resources. EPA. 2016. 
simple presencia de metano, como se ha divulgado públicamente. El estudio citado deja en evidencia que existen demasiados vacios de información, que no permiten caracterizar adecuadamente los impactos asociados a estas técnicas, por lo cual la posible asociación entre el fracking y la salud humana es un tema poco conocido. Con cerca de veinte años de desarrollo comercial intenso es una tecnología relativamente reciente, a pesar de haber iniciado su desarrollo experimental en 1947, por lo cual conocer sus efectos adversos conlleva tiempo para realizarse.

Sin embargo, entre los estudios publicados, varios trabajos realizados en Pensilvania, Estados Unidos, siendo el lugar donde se están realizando estos "experimentos naturales", tienen resultados muy llamativos. Un estudio exploró si residir cerca a los lugares con fracking mientras se estaba en embarazo tenía efectos adversos, y concluyó que entre los que viven más cerca se presenta más bajo peso al nacer y valores más bajos en un índice compuesto de salud infantil ${ }^{11}$. En otro estudio se compararon los datos de salud de los condados donde se realiza fracking con aquellos donde no se realiza, y se encontró que en los primeros habían más hospitalizaciones por neumonía entre los adultos mayores ${ }^{12}$. Más recientemente, otra investigación encontró que en las regiones con mayor densidad de pozos había más consultas médicas por problemas genitourinarios (infecciones renales, cálculos en uréteres, e infecciones del tracto urinario), principalmente entre mujeres de 20 a 64 años de edad; también se observó algo similar con hospitalizaciones cuyo motivo fueron enfermedades dérmicas ${ }^{13}$.

La preocupación por los posibles efectos de su aprovechamiento no debe ser ignorada. Es responsabilidad social de los académicos estudiar rigurosamente estos riesgos y dar respuesta desde la ciencia a los interrogantes que la sociedad se plantea como se puede vislumbrar, el tema de los efectos del fracking y la salud humana apenas se empieza a dilucidar, y exige una alta responsabilidad para los tomadores de decisión y una fuerte estrategia de vigilancia y control por parte de la sociedad. Nuevas evidencias seguirán apareciendo, y éstas deberán retroalimentar inmediatamente la política para definir si se sigue con el fracking o se detiene ante la probabilidad incrementada de daños a la salud.

\section{Referencias}

1. Gallegos TJ, Varela BA, Haines SS, Engle MA. Hydraulic fracturing water use variability in the United States and potential environmental implications, Water Resour Res. 2015; 51: 5839-5845. doi:10.1002/2015WR017278.

2. Howarth RW, Ingraffea A, Engelder T. Natural gas: Should fracking stop? Nature. 2011; 477(7364): 271-275. doi: https://doi.org/10.1038/477271a.

3. Watterson A, Dinan W. Public health and unconventional oil and gas extraction including fracking: global lessons from a Scottish government review. Int J Environ Res Public Health. 2018; 15(4): E675. doi: https://10.3390/ ijerph15040675.

4. Li L, Tan J, Wood DA, Zhao Z, Becker D, Lyu Q, et al. A review of the current status of induced seismicity monitoring for hydraulic fracturing in unconventional tight oil and gas reservoirs. Fuel. 2019; 242: 195-210. doi: https://doi.org/10.1016/j.fuel.2019.01.026.

5. Warpinski NR, Du J, Zimmer U. Measurements of hydraulic-fracture-induced seismicity in gas shales, SPE Hydraul. Fract Technol Conf., SPE 151597, The Woodlands, Texas. 2012; 1-8: doi: 10.2118/151597-PA.

6. Idrovo AJ. Terremotos, huracanes y salud humana: un llamado a estar listos. Rev Univ Ind Santander. Salud 2017; 49(4): 517-518.

7. Richburg CM, Slagley J. Noise concerns of residents living in close proximity to hydraulic fracturing sites in Southwest Pennsylvania. Public Health Nurs. 2019; 36(1): 3-10. doi: https://10.1111/phn.12540.

8. McMullin TS, Bamber AM, Bon D, Vigil DI, Van Dyke M. Exposures and health risks from volatile organic compounds in communities located near oil and gas exploration and production activities in Colorado (U.S.A.). Int J Environ Res Public Health. 2018; 15(7): E1500. doi: https://10.3390/ijerph15071500.

9. Paulik LB, Hobbie KA, Rohlman D, Smith BW, Scott RP, Kincl L, et al. Environmental and individual PAH exposures near rural natural gas extraction. Environ Pollut. 2018; 241: 397-405. doi: https://10.1016/j. envpol.2018.05.010.

10. Hu G, Kaur M, Hewage K, Sadiq R. Fuzzy clustering analysis of hydraulic fracturing additives for environmental and human health risk mitigation. Clean Techn Environ Policy. 2019; 21(1): 39-53. doi: https://doi.org/10.1007/ s10098-018-1614-3.

11. Currie J, Greenstone M, Meckel K. Hydraulic fracturing and infant health: New evidence from Pennsylvania. 
Sci Adv. 2017; 3(12): e1603021. doi: https://10.1126/sciadv.1603021.

12. Peng L, Meyerhoefer C, Chou SY. The health implications of unconventional natural gas development in Pennsylvania. Health Econ. 2018; 27(6): 956-983. doi: https://10.1002/hec.3649.

13. Denhama A, Willis M, Zavez A, Hill E. Unconventional natural gas development and hospitalizations: evidence from Pennsylvania, United States, 2003-2014. Public Health 2019; 168: doi: 17-25. https://10.1016/j. puhe.2018.11.020. 